\title{
MICRORGANISMOS PATOGÊNICOS E INDICADORES EM QUEIJO DE COALHO PRODUZIDO NO ESTADO DO CEARÁ, BRASIL
}

\author{
MARIA DE FÁTIMA BORGES* \\ TEREZINHA FEITOSA* \\ RENATA TIEKO NASSU* \\ CELLI RODRIGUES MUNIZ** \\ ÉRIKA HARDY FRANCO DE AZEVEDO*** \\ EVÂNIA ALTINA TEIXEIRA DE FIGUEIREDO****
}

\begin{abstract}
Avaliou-se a qualidade higiênico-sanitária de 43 amostras de queijo de coalho produzidas em diferentes microrregiões do estado do Ceará. Bolores e leveduras foram detectados em $100 \%$ das amostras de queijos, com contagem variando de 1,7 $\times 10^{4}$ a $1,6 \times 10^{9} \mathrm{UFC} / \mathrm{g}$. Todas as amostras apresentaram coliformes totais e fecais, com confirmação de Escherichia coli em $93,1 \%$ das mesmas. Foi verificada a presença de Estafilococos coagulase positiva em $93,1 \%$ das amostras de queijos, com contagens variando de $1,0 \times 10^{1}$ a 2,0 $\times 10^{9} \mathrm{UFC} / \mathrm{g}$. Apenas 2,3\% das amostras encontravam-se de acordo com os padrões microbiológicos vigentes para essa bactéria. A presença de Salmonella foi constatada em $34,9 \%$ das amostras de queijos. Listeria sp. foi detectada em $6,9 \%$ das amostras, com confirmação de $L$. monocytogenes em $2,3 \%$ dessas. A elevada população de bolores e leveduras (observada em 100\% das amostras de queijos de coalho) indicou deficiência nos procedimentos de higiene e sanitização das amostras, caracterizando-as como produto em condições higiênicas insatisfatórias. A alta concentração de estafilococos coagulase positiva e de coliformes fecais (aproximadamente $90 \%$ das amostras) caracterizou os queijos como produto em condições higiênico-sanitárias insatisfatórias. Queijos de coalho oriundos das cinco microrregiões do Ceará envolvidas no estudo não apresentaram segurança alimentar, visto que a maioria continha estafilococos coagulase positiva, L. monocytogenes e Salmonella. A presença dessas duas últimas bactérias permitiu classificar os queijos como produtos impróprios para consumo humano.
\end{abstract}

PALAVRAS-CHAVE: QUEIJO DE COALHO; SEGURANÇA ALIMENTAR; LATICÍNIOSQUALIDADE; Listeria monocytogenes.

* Pesquisadoras, Embrapa Agroindústria Tropical, Fortaleza, CE, Brasil (e-mail: fatima@cnpat.embrapa.br).

** Bióloga, Embrapa Agroindústria Tropical, Fortaleza, CE, Brasil.

*** Assistente de Operações, Embrapa Agroindústria Tropical, Fortaleza, CE, Brasil.

**** Professora, Departamento de Tecnologia de Alimentos, Universidade Federal do Ceará, Fortaleza, CE, Brasil. 


\section{INTRODUÇÃO}

O queijo destaca-se entre os derivados láticos pelo alto teor de proteína e outros nutrientes. Com o desenvolvimento tecnológico de sua produção surgiram, em nível nacional, muitas variedades, sendo algumas de expressão regional. O queijo de coalho, produzido no Nordeste, é um dos produtos láticos mais difundidos no estado do Ceará. Tal atividade, de importância econômica e social, é exercida por inúmeros pequenos produtores estabelecidos principalmente na zona rural.

A qualidade e a segurança alimentar do queijo de coalho limitam sua comercialização. Quase sempre são elaborados de forma artesanal e normalmente a partir de leite cru, sem os devidos cuidados de higiene ou em pequenas indústrias que não adotam Boas Práticas de Fabricação.

BASTOS et al. (2001) efetuaram inspeção em indústria produtora de queijo de coalho no Ceará e verificaram falhas em alguns procedimentos de higiene e sanificação (com reflexo na qualidade dos produtos finais). Constataram a necessidade da implantação de Boas Práticas de Fabricação para controle dos pontos críticos do processo de produção.

Vários estudos sobre a qualidade microbiológica de queijo de coalho relataram a ocorrência de microrganismos patogênicos e contagens de microrganismos deterioradores excedendo, às vezes, os limites estabelecidos pela legislação (FEITOSA et al., 2003; BASTOS et al., 2001; NASSU et al., 2000; PAIVA e CARDONHA, 1999; SANTOS et al., 1995). Dentre as bactérias patogênicas detectadas encontravam-se Staphylococcus aureus, Salmonella e Listeria monocytogenes.

Altos níveis de contaminação $\left(10^{3}\right.$ e $\left.10^{6} \mathrm{UFC} / \mathrm{g}\right)$ por $S$. aureus têm sido encontrados em queijo de coalho produzido em vários estados do Nordeste (Rio Grande do Norte, Ceará, Paraíba e Pernambuco) (FEITOSA et al., 2003; FLORENTINO e MARTINS, 1999; MENDES et al., 1999; PAIVA e CARDONHA, 1999). Esses níveis são preocupantes por não se enquadrarem nos padrões estabelecidos pelos Ministérios da Agricultura, Pecuária e Abastecimento e da Saúde, cujo máximo permitido é $10^{3} \mathrm{UFC} / \mathrm{g}$ (BRASIL, 2001 e 1996).

A presença de Salmonella em queijo de coalho tem sido relatada em algumas pesquisas (FEITOSA et al., 2003; FLORENTINO e MARTINS, 1999; PINTO et al., 1996). Sabe-se, também, que a Salmonella mantémse viável em queijo contaminado por longo tempo (MODI et al., 2001; 
BORGES, BRANDÃO e PINHEIRO, 1990). Tal fato ressalta a importância do controle de qualidade microbiológica do produto, visto que a legislação brasileira estabelece ausência dessa bactéria em alimentos (BRASIL, 2001 e 1996).

Apesar da importância da ocorrência de L. monocytogenes em produtos láticos (FEITOSA et al., 2003; PARK et al., 2002; RUDOLF e SCHERER, 2001; RYSER, 1999) existem poucos estudos sobre sua incidência em queijos produzidos no Brasil (FEITOSA et al., 2003; SILVA et al., 2003; BRANCO, 2002; SOUZA, 2002; SILVA et al., 2001; SILVA et al., 1998).

Contagens elevadas de bolores e leveduras e de bactérias do grupo coliforme também têm sido constatadas em queijos de coalho (FEITOSA et al., 2003; FLORENTINO e MARTINS, 1999), indicando produção sob condições de higiene insatisfatórias. Esses microrganismos são considerados os principais responsáveis pela deterioração de queijos.

Este trabalho teve como objetivo avaliar a ocorrência de microrganismos patogênicos e de indicadores higiênico-sanitários em queijo coalho produzido, artesanalmente, em diferentes microrregiões no estado do Ceará (Brasil).

\section{MATERIAL E MÉTODOS}

Foram analisadas 43 amostras indicativas $(n=1)$ de queijos de coalho, produzidas em 11 municípios das seguintes microrregiões do estado do Ceará: Baixo Jaguaribe, Médio Jaguaribe, Sertão Inhamuns, Sertão de Crateús e Sertão de Quixeramobim. As amostras foram coletadas aleatoriamente no local de produção e transportadas, em caixas isotérmicas contendo gelo, até o Laboratório de Microbiologia de Alimentos da Embrapa Agroindústria Tropical. Em seguida, foram avaliadas quanto ao número mais provável de coliformes totais, coliformes fecais e Escherichia coli, contagens de estafilococos coagulase positiva, contagens de bolores e leveduras e pesquisa de Salmonella sp., segundo metodologia da American Public Health Association (APHA, 2001) e de SILVA et al. (2001). A pesquisa de Listeria sp. foi realizada pelo teste ELISA e a configuração "sandwich" pelo método "TECRA Listeria Visual Imunoassay", com enriquecimento prévio em caldo de enriquecimento para Listeria e caldo Fraser (KNIGHT et al., 1996). As culturas positivas foram isoladas e avaliadas pelo método convencional para confirmação da presença de L. monocytogenes (PAGOTTO et al., 2003). 


\section{RESULTADOS E DISCUSSÃO}

A população de bolores e leveduras variou de $1,7 \times 10^{4}$ a $1,6 \times 10^{9} \mathrm{UFC} / \mathrm{g}$ (Tabela 1), evidenciando que os queijos de coalho foram processados sob condições higiênicas insatisfatórias. Tal fato compromete a qualidade e a vida-de-prateleira dos queijos, uma vez que bolores e leveduras são potenciais deterioradores de produtos láticos. FEITOSA (1985) relatou contagens de bolores e leveduras em valores que variaram de $8,6 \times 10^{3} \mathrm{a}$ $3,2 \times 10^{6} \mathrm{UFC} / \mathrm{g}$ em amostras de queijo de coalho produzidos no Ceará. FEITOSA et al. (2003) também constataram elevadas contagens desses microrganismos $\left(1,9 \times 10^{4}\right.$ a $\left.4,8 \times 10^{8} \mathrm{UFC} / \mathrm{g}\right)$ em queijo de coalho produzido em diferentes microrregiões do Rio Grande do Norte.

Todas as amostras de queijos avaliadas apresentaram coliformes totais e coliformes fecais, sendo confirmada a presença de Escherichia coli em $93 \%$ das amostras (Tabela 1). Em relação a coliformes fecais observouse que $74,4 \%$ das amostras continham níveis superiores aos padrões estabelecidos pela legislação vigente (BRASIL, 2001, 1996). Número elevado desses microrganismos indica alto nível de contaminação fecal, normalmente decorrente da qualidade da matéria-prima ou do processamento dos queijos sob condições inadequadas de higiene. A ocorrência de coliformes fecais em queijo de coalho em níveis superiores aos permitidos pela legislação tem sido relatada em vários estudos. No Ceará, FEITOSA (1985) constatou a presença de coliformes totais e fecais em $100 \%$ das amostras de queijo de coalho artesanal analisadas. No Rio Grande do Norte, FEITOSA et al. (2003) observaram coliformes fecais em $36 \%$ das amostras de queijo de coalho produzido em diferentes microrregiões do Estado. Na Paraíba, FLORENTINO e MARTINS (1999) detectaram índice elevado de coliformes fecais $\left(9,8 \times 10^{2}\right.$ a $1,2 \times 10^{5} \mathrm{NMP} /$ g) em queijo artesanal. Em Pernambuco, PAIVA e CARDONHA (1999) também constataram elevadas contagens de coliformes fecais na maioria das amostras de queijo de coalho comercializado em Recife (procedentes de 15 municípios).

A ocorrência de estafilococos coagulase positiva foi observada em $93,1 \%$ das amostras de queijo de coalho (Tabela 1), sendo que apenas 2,3\% das mesmas enquadraram-se nos padrões microbiológicos vigentes para esta bactéria $\left(5,0 \times 10^{3} \mathrm{UFC} / \mathrm{g}\right)$. Assim, 90,8\% dos queijos foram classificados como produto em condições higiênico-sanitárias insatisfatórias. Concentrações superiores a $10^{5}$ células/g podem propiciar a produção de enterotoxinas (FORSYTHE, 2000) e oferecer risco potencial à saúde do consumidor. A prevalência de $S$. aureus como agente etiológico 
da mastite bovina, sua ubiqüidade na natureza e o baixo nível socioeconômico dos ordenhadores (muitas vezes portadores assintomáticos e com hábitos higiênicos não-adequados) são fatores que favorecem a contaminação dos queijos (ADAMS e MOSS, 2000; GOMES e GALLO, 1995).

\section{TABELA 1 - CARACTERIZAÇÃO MICROBIOLÓGICA DE QUEIJO COALHO PRODUZIDO EM DIFERENTES MICRORREGIÕES DO ESTADO DO CEARÁ}

\begin{tabular}{|c|c|c|c|}
\hline Microrganismos & Contagens & $\begin{array}{c}\mathrm{N} \text { de } \\
\text { amostras }\end{array}$ & $\%$ \\
\hline Bolores e leveduras (UFC/g) & $1,7 \times 10^{4}$ a $1,6 \times 10^{9}$ & 43 & 100 \\
\hline \multirow{2}{*}{ Coliformes totais (NMP/g) } & 28 a 450 & 8 & 18,6 \\
\hline & $451 \mathrm{a} \geq 2400$ & 35 & 81,4 \\
\hline \multirow{3}{*}{ Coliformes fecais (NMP/g) } & 11 a 500 & 11 & 25,6 \\
\hline & $501 \mathrm{a} \geq 2.400$ & 32 & 74,4 \\
\hline & $<3$ & 3 & 7,0 \\
\hline \multirow[t]{3}{*}{ Escherichia coli (NMP/g) } & 3 a 240 & 36 & 83,7 \\
\hline & $1.100 \mathrm{a} \geq 2.400$ & 4 & 9,3 \\
\hline & $<10$ & 3 & 6,9 \\
\hline \multirow[t]{2}{*}{$\begin{array}{l}\text { Estafilococos coagulase } \\
\text { positiva (UFC/g) }\end{array}$} & $1,0 \times 10^{3}$ & 1 & 2,3 \\
\hline & $3,0 \times 10^{4}$ a $2,0 \times 10^{9}$ & 39 & 90,8 \\
\hline \multirow{2}{*}{ Salmonella sp. (em $25 \mathrm{~g}$ ) } & AusOfrcia & 28 & 65,1 \\
\hline & Presen a & 15 & 34,9 \\
\hline \multirow{2}{*}{ Listeria sp.(em $25 \mathrm{~g})$} & AusCfrcia & 40 & 93,1 \\
\hline & Presen a & 3 & 6,9 \\
\hline \multirow{2}{*}{ L. monocytogenes (em $25 \mathrm{~g}$ ) } & AusCfrcia & 42 & 97,7 \\
\hline & Presen a & 1 & 2,3 \\
\hline
\end{tabular}

FEITOSA (1985) observou a presença de S. aureus em 100\% das amostras de queijo de coalho produzidos no Ceará. Alto índice de ocorrência desse patógeno em queijo de coalho também foi relatado em queijos produzidos nos estados de Pernambuco (MENDES et al., 1999), da Paraíba (FLORENTINO e MARTINS, 1999) e do Rio Grande do Norte (FEITOSA et al., 2003; PAIVA e CARDONHA, 1999). 
Salmonella foi detectada em $34,9 \%$ das amostras de queijo de coalho (Tabela 1). A ingestão de Salmonella causa infecção alimentar e a legislação brasileira não permite sua presença em alimentos. Dessa maneira, os queijos analisados foram classificados como produtos impróprios para consumo. FEITOSA et al. (2003) relataram a presença de Salmonella em $9 \%$ dos queijos analisados, em diferentes microrregiões do estado do Rio Grande do Norte, enquanto que FLORENTINO e MARTINS (1999) observaram a presença dessa bactéria em $30 \%$ das amostras de queijo artesanal produzido em várias regiões do estado da Paraíba. Em queijo de coalho comercializado em Recife, MENDES et al. (1999) verificaram a presença de Salmonella em 73,3\% das amostras oriundas de 15 municípios de Pernambuco.

Listeria sp. foi detectada em 6,9\% das amostras de queijos coalho pelo teste ELISA, com confirmação de L. monocytogenes em 2,3\% dessas pelo método convencional (Tabela 1). Como a presença desse patógeno não é permitida pela legislação, os queijos foram classificados como impróprios para o consumo. SOUZA (2002) também constatou baixa incidência $(1,4 \%)$ de $L$. monocytogenes em amostras de queijo coalho artesanal comercializado a temperatura ambiente em Fortaleza-CE. Por outro lado, em queijo de coalho refrigerado, produzido industrialmente no Ceará, BRANCO (2002) detectou L. monocytogenes em 19\% das amostras de queijo avaliadas.

\section{CONCLUSÃO}

Os queijos de coalho oriundos das seis microrregiões do Ceará envolvidos no estudo não apresentam segurança alimentar, visto que em média 74\% das amostras apresentaram coliformes fecais, $90 \%$ estafilococos coagulase positiva, 35\% presença de Salmonella e $2 \%$ L. monocytogenes. Além disso, a elevada população de bolores e leveduras detectada em $100 \%$ dos queijos indica deficiência nos procedimentos de higiene durante o processamento. Nessas condições, os queijos foram classificados como impróprios para o consumo humano, conforme padrões microbiológicos da legislação brasileira vigente.

\section{Abstract}

PATHOGENIC AND INDICATOR MICROORGANISMS ISOLATED FROM “COALHO”CHEESE PRODUCED IN THE CEARÁ STATE, BRAZIL

The hygienic-sanitary quality of 43 "coalho" cheese samples produced in different regions 
of the Ceará state was evaluated. Yeasts and molds were detected in $100 \%$ of the samples, varying from $1.7 \times 10^{4}$ to $1.6 \times 10^{9} \mathrm{CFU} / \mathrm{g}$. All of the samples presented total and faecal coliforms with confirmation of Escherichia coli in $93.1 \%$ of the samples. Positive coagulase staphylococci were observed in $93,1 \%$ of the samples with countings ranging from $1.0 \times 10^{1}$ to $2.0 \times 10^{9} \mathrm{CFU} / \mathrm{g}$. Only $2.3 \%$ of the samples were in accordance with the actual microbiological patterns for this bacteria. Presence of Salmonella was confirmed in $34.9 \%$ of the cheese samples. Listeria sp. was detected in $6.9 \%$ of the samples and posterior confirmation of $L$. monocytogenes in $2.3 \%$ of the samples. The elevated population of yeasts and molds (observed in $100 \%$ of the samples) indicated deficiency in the hygiene and sanitation proceedings becoming characterized as "product at unsatisfactory hygienic conditions". The high population of positive coagulase staphylococci characterized the cheeses as "product at unsatisfactory hygienic and sanitary conditions". "Coalho" cheeses originated from five regions of Ceará state involved in this study did not present food safety, mainly because of the presence of positive coagulase staphylococci, L. monocytogenes and Salmonella. The presence of the two last bacteria permitted to classify the cheeses as inappropriate to the human consumption.

KEY-WORDS: COALHO CHEESE; FOOD SAFETY; DAIRY-PRODUCTS-QUALITY; Listeria monocytogenes.

\section{REFERÊNCIAS}

1 ADAMS, M.R.; MOSS, Y.M.O. Food microbiology. $2^{\text {nd }}$ ed. Cambridge: The Royal Society of Chemistry, 2000. p. 258-263.

2 APHA. American Public Health Association. Compendium of methods for the microbiological examination of foods. $4^{\text {th }}$ ed. Washington, 2001. 676 p.

3 BASTOS, M. do S.R.; NASSU, R.T.; BORGES, M. de F.; SILVA, J.B. Inspeção em uma indústria produtora de queijo tipo coalho no Estado do Ceará, visando a implantação das Boas Práticas de Fabricação. Revista do Instituto de Laticínios Cândido Tostes, Juiz de Fora, v. 57, n. 321, p. 130136, jul./ago. 2001.

4 BORGES, M. de F.; BRANDÃO, S.C.C.; PINHEIRO, A.J.R. Sobrevivência de Salmonella em queijo minas padronizado durante a maturação. Revista de Microbiologia, São Paulo, v. 21, n. 3, p. 276-281, jul./set. 1990.

5 BRANCO, M.A. de A.C. Incidência de Listeria monocytogenes em queijo de coalho refrigerado produzido industrialmente. Fortaleza. 2002. 63 p. Dissertação (Mestrado em Tecnologia de Alimentos) - Departamento de Tecnologia de Alimentos, UFC.

6 BRASIL. Ministério da Agricultura. Portaria no 146, de 07/03/1996. 
Regulamento técnico de identidade e qualidade de queijos. Diário Oficial [da] República Federativa do Brasil, Brasília, 11/03/1996. p. 3977-3978.

7 BRASIL. Ministério da Saúde. Agência Nacional de Vigilância Sanitária. Resolução RDC no 12, de 02/01/2001. Regulamento técnico sobre os padrões microbiológicos para alimentos. Diário Oficial [da] República Federativa do Brasil, Brasília, 02/01/2001. p. 1-54.

8 FEITOSA, T. Estudos tecnológicos, físico-químicos, microbiológicos e sensoriais do queijo de coalho do estado do Ceará. Fortaleza. 1985. 96 p. Dissertação (Mestrado em Tecnologia de Alimentos) - Departamento de Tecnologia de Alimentos, UFC.

9 FEITOSA, T.; BORGES, M. de F.; NASSU, R.T.; AZEVEDO, E.H. de F.; MUNIZ, C.R. Pesquisa de salmonella sp., Listeria sp. e microrganismos indicadores higiênico-sanitários em queijos produzidos no Estado do Rio Grande do Norte. Ciência e Tecnologia de Alimentos, Campinas, v. 23, n. 3, set./dez. 2003. (No prelo).

10 FLORENTINO, E.S.; MARTINS, R.S. Características microbiológicas do "queijo de coalho" produzido no Estado da Paraíba. Revista Higiene Alimentar, São Paulo, v. 13, n. 59, p. 43-48, jan./fev. 1999.

11 FORSYTHE, S.J. The microbiology of safe food. London: Blackwell Science, 2000. p. 155-201.

12 GOMES, H. de A.; GALLO, G. R. Ocorrência de Staphylococcus aureus e produção de enterotoxinas por linhagens isoladas a partir de leite cru, leite pasteurizado tipo $\mathrm{C}$ e queijo minas frescal comercializados em Piracicaba - SP. Ciência e Tecnologia de Alimentos, Campinas, v. 15, n. 2, p. 158-161, jul./dez. 1995.

13 KNIGHT, M.T.; NEWMAN, M.C.; BENZINGER Jr, M.J.; AGIN, J.R.; ASH, M.; SIMS, P.; HUCHES, D. Tecra Listeria visual immunoassay (TLVIA) for detection of Listeria in foods; collaborative study. Journal of AOAC International, v. 79, n 5, p. 1083-1094, 1996.

14 MENDES, E.S.; LIMA, E.C.; NUMERIANO, A.K.M.; COELHO, M.I.S. Staphylococcus aureus, Salmonella sp. e coliformes em queijos de "coalho" comercializados em Recife. Higiene Alimentar, São Paulo, v. 13, n. 66/67, p. 122-126, nov./dez. 1999.

15 MODI, R.; HIRVI, Y.; HILL, A.; GRIFFITHS, M.W. Effect of phage on survival of Salmonella enteritidis during manufacture and storage of cheddar 
cheese made from raw and pasteurized milk. Journal Food Protection, Des Moines, v. 64, n. 7, p. 927-933, 2001.

16 NASSU, R.T.; MOREIRA, C.G.; ROCHA, R.G. de A.; FEITOSA, T.; BORGES, M. de F.; MACEDO, A.A.M. Diagnóstico das condições de processamento e qualidade microbiológica de produtos regionais derivados do leite produzido no Estado do Rio Grande do Norte. Revista do Instituto de Laticínios Cândido Tostes, Juiz de Fora, v. 55, n. 315, p. 21-126, jul./ago. 2000.

17 PAGOTTO, F.; DALEY, E.; FARBER, J.; WARBURTON, D. Health products and food branch Ottawa: isolation of Listeria monocytogenes from all foods and environmental samples. Government of Canada, Janeiro 2001. Disponível em: <http:www.hc-sc.ca/food-aliment>. Acesso em 27 jan. 2003.

18 PAIVA, M.S.D.; CARDONHA, A. M. S. Queijo de coalho artesanal e industrializado produzidos no Rio Grande do Norte: estudo comparativo da qualidade microbiológica. Revista Higiene Alimentar, São Paulo, v. 13, n. 61, p. 33-37, jan./fev. 1999.

19 PARK, S.; SPAHR, U.; JEMMI, T.; SALMAN, M.D. Risk factors for L. monocytogenes contamination of dairy products in Switzerland, 19901999. Preventive Veterinary Medicine, Fort Collins, v. 53, n. 1, p. 55-65, jan., 2002.

20 PINTO, P. S. A.; GERMANO, M. I. S.; GERMANO, P. M. L. Queijo minas: problema emergente de Saúde Pública. Revista Higiene Alimentar, São Paulo, v. 10, n. 44, p. 22-27, jan./fev. 1996.

21 RUDOLF, M.; SCHERER, S. High incidence of Listeria monocytogenes in European red smear cheese. International Journal of Food Microbiology, Amsterdam, v. 63, p. 91-98, jan. 2001.

22 RYSER, E.T. Incidence and behavior of Listeria monocytogenes in cheese and other fermented dairy products. In: RYSER, E. T.; MARTH, E. H. (Ed.) Listeria, listeriosis and food safety. $2^{\text {nd }}$ ed. New York: Marcel Dekker, 1999. Cap. 12, p. 411-503.

23 SANTOS, F. A.; NOGUEIRA, N. A. P.; CUNHA, G. M. A. Aspectos microbiológicos do queijo tipo coalho comercializado em Fortaleza Ceará. Boletim do Centro de Pesquisa e Processamento de Alimentos, Curitiba, v. 13, n. 1, p. 31-361, jan./jul. 1995. 
24 SILVA, M.C.D.; HOFER, E.; TIBANA, A. Incidence of Listeria monocytogenes in cheese produced in Rio de Janeiro, Brazil. Journal of Food Protection, Des Moines, v. 61, n. 3, p. 354-356, mar. 1998.

25 SILVA, M.C.D.; DESTRO, M.T.; HOFER, E.; TIBANA, A. Characterization and evaluation of some virulence markers of Listeria monocytogenes strains isolated from Brazilian cheeses using molecular, biochemical and serotyping techniques. International Journal of Food Microbiology, Amsterdam, v. 63, p. 275-280, jan. 2001.

26 SILVA, I.M.M.; ALMEIDA, R.C.C.; ALVES, M.A.O.; ALMEIDA, P.F. Occurrence of Listeria spp. in critical control points and the environment of Minas Frescal cheese processing. International Journal of Food Microbiology, Amsterdam, v. 81, n. 2, p. 241-248, Feb. 2003.

27 SILVA, N.; JUNQUEIRA, V.C.A.; SILVEIRA, N.F.A. Manual de métodos de análise microbiológica de alimentos. 2. ed. São Paulo: Livraria Varela, 2001. 229 p.

28 SOUZA, R.A. Incidência de L. monocytogenes em queijo tipo coalho artesanal comercializado a temperatura ambiente em Fortaleza - CE. Fortaleza, 2002. 78 p. Dissertação (Mestrado em Tecnologia de Alimentos) - Departamento de Tecnologia de Alimentos, UFC.

\section{AGRADECIMENTOS}

Ao Banco Mundial/PRODETAB pelo financiamento do projeto. 